\title{
Universal response of quantum systems with chaotic dynamics
}

\author{
Diego A. Wisniacki ${ }^{1}$, Natalia Ares ${ }^{1}$, and Eduardo G. Vergini ${ }^{2,3}$ \\ ${ }^{1}$ Departamento de Física, FCEyN, UBA, Ciudad Universitaria, Buenos Aires C1428EGA, Argentina \\ ${ }^{2}$ Departamento de Física, Comisión Nacional de Energía Atómica., Av. Libertador 8250, Buenos Aires, Argentina \\ 3 Departamento de Física, E.T.S.I. Agrónomos, Universidad Politécnica de Madrid, 28040-Madrid, Spain
}

(Dated: October 9, 2018)

\begin{abstract}
The prediction of the response of a closed system to external perturbations is one of the central problems in quantum mechanics, and in this respect, the local density of states (LDOS) provides a deep description of such a response. The LDOS is the distribution of the overlaps squared connecting the set of eigenfunctions with the perturbed one. Here, we show that in the case of closed systems with classically chaotic dynamics, the LDOS is a Breit-Wigner distribution under very general perturbations of arbitrary high intensity. Consequently, we derive a semiclassical expression for the width of the LDOS which is shown to be very accurate for paradigmatic systems of quantum chaos. This work demonstrates the universal response of quantum systems with classically chaotic dynamics.
\end{abstract}

PACS numbers: 05.45.Mt; 05.45.Ac; 05.45.Pq

The action of a perturbation on eigenfrequencies and eigenfunctions of a quantum system has been a subject of paramount importance since the beginning of quantum theory. Its understanding is at the heart of fundamental problems of quantum mechanics like dissipation, phase transition or irreversibility. The usual perturbation theory is a good starting point to describes successfully this effect when the perturbation is small. However, approximated theories usually fail for strong perturbations and highly demanding computational methods are needed to describe characteristics of the perturbed system.

The local density of states (LDOS) or strenght function is a widely studied magnitude to characterize the effect of perturbations on quantum systems and has been extensively computed for different systems and perturbations [1-5]. The LDOS is a distribution of the overlaps squared between the unperturbed and perturbed eigenstates. Let us consider a chaotic one parameter dependent Hamiltonian $H(x)$, and its quantum counterpart $\hat{H}(x)$ with eigenfrequencies $\omega_{j}(x)$ and eigenstates $|j(x)\rangle$. Then, the LDOS of an eigenstate $\left|i\left(x_{0}\right)\right\rangle$ (that we call unperturbed) is given by,

$$
\rho_{i}(\omega, \delta x)=\sum_{j}\left|\left\langle j(x) \mid i\left(x_{0}\right)\right\rangle\right|^{2} \delta\left(\omega-\omega_{i j}\right),
$$

with $\delta x=x-x_{0}$ and $\omega_{i j}=\omega_{j}(x)-\omega_{i}\left(x_{0}\right)$. Furthermore, to avoid a dependence on the particular characteristics of the state $\left|i\left(x_{0}\right)\right\rangle$, an average over $n$ unperturbed states in a small frequency window is performed

$$
\bar{\rho}(\omega, \delta x)=\frac{1}{n} \sum \rho_{i}(\omega, \delta x) .
$$

The LDOS is related with important measures of irreversibility and sensitivity to perturbations in quantum systems as the survival probability and the Loschmidt echo (LE) [68]. In fact, the LDOS is the Fourier tranform of the survival probability [9] and its width gives the decay rate of the LE for a small enough strength of the perturbation [7, 8]. In this letter these relations are exploited to show that LDOS has Lorentzian shape, usually called the Breit-Wigner distribution, under very general perturbations of arbitrary high intensity. Moreover, we derive a semiclassical expression for the width of the LDOS, $\sigma_{s c}$, in chaotic systems. The derived expression only depends on the perturbation, while the properties of the system are taken into account through a uniform measure in phase space. Although $\sigma_{s c}$ is derived for local perturbations we show that it also works in the case of global perturbations as a consequence of the Lorentzian character of the LDOS, and the requirement of statistical independence between perturbed and unperturbed eigenfunction sets. Of course, such a requirement imposes restrictions on the admitted perturbations and we discuss this point at the end of the letter. We test the ability of $\sigma_{s c}$ to predict the width of the LDOS in perturbed cat maps and the Bunimovich stadium billiard with boundary deformations, observing that it works very well in both systems, even for strong perturbations far away from the Fermi Golden Rule regime. These results demonstrate for the first time the universal response of quantum chaotic systems to perturbations of classical nature.

The Fourier transform of Eq. (1) is given by,

$$
\mathscr{F}[\bar{\rho}](t, \delta x)=\frac{1}{n} \sum e^{-\mathrm{i} \omega_{i}\left(x_{0}\right) t}\left\langle i\left(x_{0}\right)\left|e^{\mathrm{i} \hat{H}(x) t / \hbar}\right| i\left(x_{0}\right)\right\rangle,
$$

where the sum runs over the amplitude fidelity of eigenstates (whose square modulus is the survival probability). Let us evaluate the previous sum semiclassically. Vanícek has proposed an approximation of the amplitude fidelity, named the dephasing representation [11], by assuming a classically small perturbation in such a way that the shadowing theorem [10] is valid. Using such an approximation, Eq. (2) leads to,

$$
\mathscr{F}[\bar{\rho}](t, \delta x) \approx \int d q d p W(q, p) \exp \left[-\mathrm{i} \Delta S_{t}(q, p, \delta x) / \hbar\right],
$$

where $\Delta S_{t}(q, p, \delta x)$ is the action difference evaluated along the unperturbed orbit starting at $(q, p)$ that evolves a time $t$. Moreover, $W(q, p)=(1 / n) \sum W_{i}(q, p)$, with $W_{i}(q, p)$ being the Wigner function of $\left|i\left(x_{0}\right)\right\rangle$. In chaotic systems, $W(q, p)$ reduces to a uniform distribution.

In the case of local perturbations, the right hand side of Eq. (3) has been evaluated on a Poincaré surface of section by Goussev et al. [12] resulting,

$$
\mathscr{F}[\bar{\rho}](t, \delta x) \approx e^{-\gamma|t|},
$$




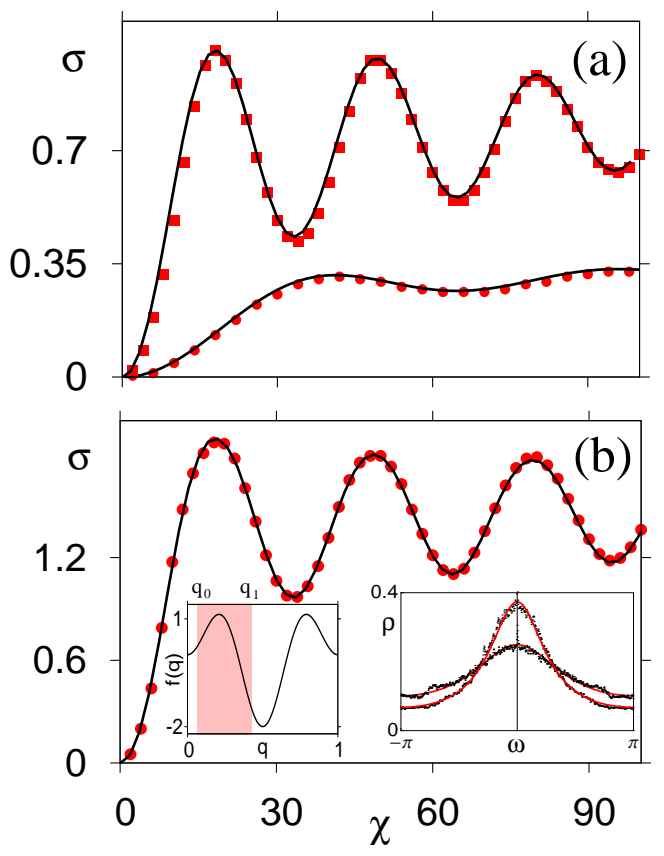

FIG. 1: (Color online) Width $\sigma$ of the LDOS as a function of the scaled perturbation strength $\chi=N \delta k$, for a local perturbation. Solid symbols correspond to the quantum case and solid lines to the semiclassical calculation $\sigma_{s c}^{(p)}$. We use $N=800$ and $q_{0}=0.01$. In panel (a) we use a width $\beta=0.2$ (circles) and $\beta=0.4$ (squares). In panel (b) $\beta=0.7$. Left inset: Schematic figure showing the used local perturbation. The scaled shear $[f(q) \equiv 2 \pi \varepsilon(q, k) / k$, (with $k$ the strength of the perturbation)] is plotted as a function of $q$. The limits of the perturbed region are indicated with $q_{0}$ and $q_{1}$, being $\beta=q_{1}-q_{0}$ its width. Right inset: The LDOS $\rho$ for $\beta=0.7$, with $\chi=78.4$ and 89.6. In solid (red) lines we plot periodized Lorentzian functions with the corresponding widths.

with

$$
\gamma=\eta\left(1-\Re\left\langle e^{-\mathrm{i} \Delta S(q, p, \delta x) / \hbar}\right\rangle\right) .
$$

The average is evaluated on the region of surface of section where the local perturbation acts, and $\Delta S(q, p, \delta x)$ is the action difference after one step on the surface of section. For numerical comparison we consider a rectangular region,

$$
\left\langle e^{-\mathrm{i} \Delta S(q, p, \delta x) / \hbar}\right\rangle=\frac{1}{\alpha} \int_{p_{1}}^{p_{2}} \int_{q_{1}}^{q_{2}} e^{-\mathrm{i} \Delta S(q, p, \delta x) / \hbar} d q d p,
$$

where $p_{1}, p_{2}, q_{1}$ and $q_{2}$ are the limits of the perturbed region with area $\alpha=\left(p_{2}-p_{1}\right)\left(q_{2}-q_{1}\right)$. Moreover, $\eta$, named the classical decay rate in Ref. [12], is the probability to reach the perturbed region per unit time,

$$
\eta=\frac{\alpha}{\tau \mathscr{A}}
$$

where $\mathscr{A}$ is the area of the Poincaré surface of section and $\tau$ is the mean mapping time.
The inverse Fourier tranform of Eq. (4) is a Lorentzian function, the so-called Breit-Wigner distribution,

$$
\bar{\rho}(\omega, \delta x) \approx L(\gamma, \omega)=\frac{\gamma}{\pi\left(\omega^{2}+\gamma^{2}\right)},
$$

and we define its width as half the distance around the mean value that contains the $70 \%$ of the probability (actually, this value is only relevant for the numerical computation). Then, the semiclassical approximation of the width results,

$$
\sigma_{s c}=\tan \left(0.7 \frac{\pi}{2}\right) \gamma \approx 1.963 \gamma .
$$

We stress out that the semiclassical approximation was derived in the limit of $\alpha \rightarrow 0$. However, our final expression (Eq. (9) can be extended to arbitrary values of $\alpha$ as a result of the Lorentzian character of the LDOS and the property of short range correlation of chaotic eigenfunctions. To clarify this point, let us consider the following basis sets: $\left\{\left|i^{(0)}\right\rangle\right\}$ be the set of unperturbed eigenfunctions, $\left\{\left|i^{(1)}\right\rangle\right\}$ be the set resulting after applying a local perturbation over an infinitesimal region $\delta \alpha_{1}$, and $\left\{\left|i^{(2)}\right\rangle\right\}$ be the set resulting after applying the perturbation $\delta \alpha_{2}$ to the previous system (the one with the perturbation $\delta \alpha_{1}$ ). For $\delta \alpha_{1}$ and $\delta \alpha_{2}$ being perturbations over disjointed regions of phase space, their corresponding LDOS should be statistical independent because chaotic eigenfunctions have correlations of short range in phase space. Therefore by assuming independence, the LDOS connecting the first and third basis sets is simply derived by convoluting the previous ones,

$$
\int L\left(\delta \gamma_{1}, \omega_{1}\right) L\left(\delta \gamma_{2}, \omega-\omega_{1}\right) \mathrm{d} \omega_{1}=L\left(\delta \gamma_{1}+\delta \gamma_{2}, \omega\right) .
$$

The new LDOS is also a Lorentzian function, with $\delta \gamma_{1}\left(\delta \gamma_{2}\right)$ being the parameter corresponding to the first (second) perturbation. Then, by using Eqs. (5), (6) and (7) it is easy to see that $\delta \gamma_{1}+\delta \gamma_{2}$ is just the parameter resulting from the perturbation $\delta \alpha_{1}+\delta \alpha_{2}$. Following this procedure, we can now add a perturbation $\delta \alpha_{3}$ and so on up to fill a finite region $\alpha$. We would like to stress that even though the property of statistical independence is very reasonable, there are exceptional perturbations where such a property is not satisfied; see the end of the letter for a discussion of this point.

Let us show the power of the semiclassical approximation to describe the LDOS in quantum maps, where the mapping time is fixed to $\tau=1$, the phase space is a torus of area $\mathscr{A}=1$, and the Hilbert space has finite dimension $N$ (with $2 \pi \hbar=1 / N$ ). We consider the cat map, a canonical example in classical and quantum chaos studies, perturbed with a nonlinear shear in momentum,

$$
\begin{aligned}
& q^{\prime}=2 q+p \\
& p^{\prime}=3 q+2 p+\varepsilon(q, k)(\bmod 1)
\end{aligned}
$$

where $\varepsilon(q, k)=(k / 2 \pi)[\cos (2 \pi q)-\cos (4 \pi q)]$, with $k$ being the strength of the perturbation. The action difference for one iteration of the map is given by $\Delta S(q, \delta k)=$ $\left(\delta k / 4 \pi^{2}\right)[\sin (2 \pi q)-\sin (4 \pi q) / 2][13]$. 


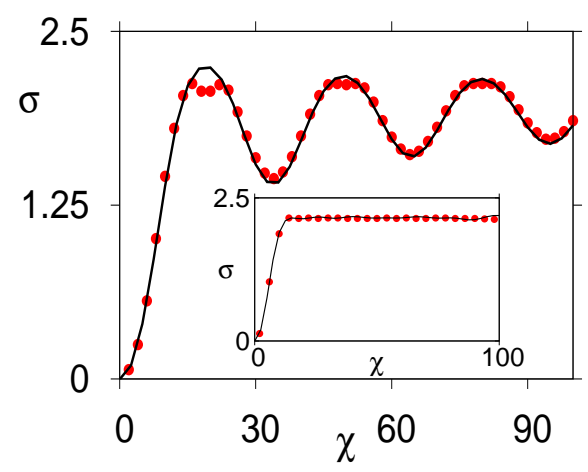

FIG. 2: Idem Fig. 1 for global perturbations. The perturbation in the main plot is the same as in Fig. 1. For the inset, the perturbation is a shear in momentum and position (see text for details).

For local perturbations [14], the shear $\varepsilon(q, k)$ is only applied to a $q$ window from $q_{0}$ to $q_{1}$, with width $\beta=q_{1}-q_{0}$ [see left inset in Fig.1]; so, $\alpha=\beta$. Furthermore, we take into account the fact that the spectrum of the cat map is periodic because of a compact phase space. This periodicity changes the form of the LDOS because the probability that leaves from one border returns to the other. By assuming no correlation between the existing and returning probabilities, the LDOS transforms into a periodized Lorentzian function

$$
L^{(p)}(\gamma, \omega)=\sum_{j=-\infty}^{\infty} L(\gamma, \omega-2 \pi j / \tau)
$$

with $\omega \tau$ being the variable that specify the spectrum of eigenphases. This distribution provides a new relation between the width of the distribution and $\gamma$, whose first correction with respect to Eq. (9) is $\left.\sigma_{s c}^{(p)} \simeq \sigma_{s c}\left[1-\left(\sigma_{s c} / \pi\right)^{2}\right)\right]$. On the other hand, a numerical computation reveals a linear term which should be related to correlations between the existing and returning probabilities. We obtain the following estimate,

$$
\sigma_{s c}^{(p)} \approx \sigma_{s c}\left[1+0.24 \sigma_{s c}-\left(\sigma_{s c} / \pi\right)^{2}\right],
$$

by fitting the linear term to the numerical data.

Fig 1 shows the width, $\sigma$, of the LDOS and its semiclassical approximation, $\sigma_{s c}^{(p)}$, for three different windows in positions where the perturbation is applied. We plot the width as a function of the scaled perturbation strength, $\chi=N \delta k$, in such a way that figures are insensitive to $N$. As it can be seen the semiclassical approximation works very well. Moreover, the right inset of Fig 1 (b) shows that the LDOS is a periodized Lorentzian function even for strong perturbations far away from the Fermi Golden Rule regime, which is identified with the quadratic behavior close to the origin.

Fig. 2 compares the width of the LDOS with $\sigma_{s c}^{(p)}$ in the case of global perturbations $(\alpha=1)$. The perturbation in the main panel is the one used previously while in the inset, we use the same shear in momentum plus the following shear in position: $\bar{\varepsilon}(p, k)=-(k / 2 \pi)[\sin (6 \pi p) / 3+\cos (4 \pi p) / 2]$.

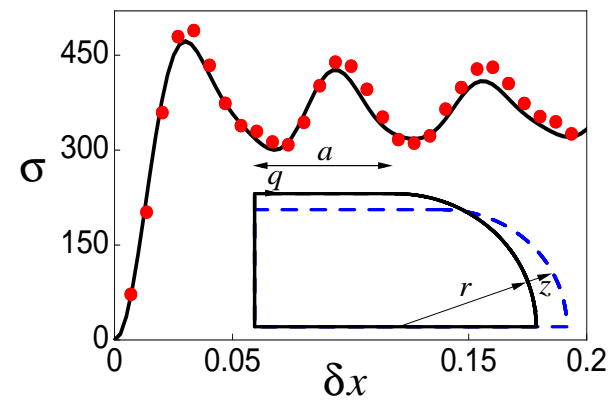

FIG. 3: $\sigma$ as a function of the perturbation strenght $\delta x$ for the stadium billiard in the region of wave number around 200 (solid circles). The semiclassical approximation $\sigma_{s c}$ using Eq. (9) is plotted with solid line. Inset: two Bunimovich stadium billiards with different shape parameter; the perturbed system is indicated in dashed line.

In the latter the action difference is, $\Delta S(q, p, \delta k)=$ $\left(\delta k / 4 \pi^{2}\right)[\sin (2 \pi q)-\sin (4 \pi q) / 2+\cos (6 \pi p)-\sin (4 \pi p) / 2]$. Differences near the peaks at $\chi \approx 20$ and $\chi \approx 50$ are related to the poor accuracy of Eq. (10) close to the saturation, which is given by the width of the uniform distribution, $0.7 \pi \approx 2.20$.

To further demonstrate the power of the proposed semiclassical approximation in a realistic system, we consider the desymmetrized Bunimovich stadium billiard with radius $r$ and straight line of lenght $a$. This system is fully chaotic [15] and has great theoretical and experimental relevance. The billiard is perturbed by the boundary deformation displayed in the inset of Fig 3 . The area of the billiard is fixed to the value $1+\pi / 4$, so the boundary only depends on the shape parameter $x=a / r$. The boundary deformations are parametrized by $\mathbf{r}(q, \delta x)=\mathbf{r}_{\mathbf{0}}(q)+z(q, \delta x) \mathbf{n}$, where $q$ is a coordinate along the unperturbed boundary $\mathscr{C}, \mathbf{r}_{\mathbf{0}}(q)$ defines $\mathscr{C}$ and $\mathbf{n}$ is the outward normal unit vector to $\mathscr{C}$ at $\mathbf{r}_{0}(q)$ (an explicit expression for $z(q, \delta x)$ is provided in Ref. [16]). We consider the usual Birkhoff coordinates to describe the classical dynamics of the particle; that is, the variables $q$ and $p=\sin (\theta)$, with $\theta$ the impinging angle with $\mathbf{n}$. To compute $\sigma_{s c}$, the action difference between the unperturbed and perturbed orbit results in [12],

$$
\Delta S(q, \theta, \delta x)=|\mathbf{p}| \Delta L=2|\mathbf{p}| z(q, \delta x) \cos (\theta),
$$

where $\Delta L$ is the lenght difference between the unperturbed and perturbed orbits, and $\mathbf{p}$ is the momentum of the particle. The mean time between bounces with the boundary is given by $\tau=m \pi A /(|\mathbf{p}| \mathscr{P})$ [17], with $m$ the mass of the particle, $A$ the area of the billiard and $\mathscr{P}$ its perimeter. Then, the decay rate results in $\eta=|\mathbf{p}| \beta /(m \pi A)$, with $\beta$ the width of the perturbed region[12]; for the selected perturbation, $\beta=\mathscr{P}$. Fig. 3 compares the numerical and semiclassical calculations; $\hbar=1$ and $m=1 / 2$ are used. The width computed with the exact eigenstates is plotted with full circles and the semiclassical aproximation, $\sigma_{s c}$, is plotted in full line. The calculations displayed in Fig. 3 were performed around the wave number 200 , where the eigenstates of the billiard were computed using the scaling method [18]. The agreement between the quantum and the semiclassical calculation is excellent. We notice that while the full quantum computation of $\sigma$ in Fig. 3 is very time 
consuming $\left[t \approx 7 \times 10^{7} \mathrm{seg}\right.$ in an CPU Intel Core 26400$]$, the semiclassical calculation is a simple two variable integral.

One final point is to discuss the character of the perturbation in order to satisfy the used property of statistical independence between the perturbed and unperturbed eigenfunction sets. Let us consider as an extreme example a chaotic Hamiltonian of the form kinetic plus potential energy, and where the perturbed Hamiltonian is obtained by a displacement of the potential energy. In this situation, the two systems have the same spectrum and the corresponding eigenfunctions are connected by the displacement. So, the two sets are strongly correlated and the LDOS does not satisfy the Breit-Wigner distribution; see for instance Ref. [19] where this type of perturbations is analyzed. From the classical point of view, we notice that the dynamics and in particular, the structure of periodic orbits are not affected at all by the perturbation. In this context, the question that immediately arises is how the perturbation has to modify the dynamics in order to guarantee the required independence. We develop the answer within the short periodic orbit approach [20], where the eigenfunctions of a chaotic system are described in a scar function basis set [21]; these wave functions are supported by the shortest periodic orbits of the system, with periods up to the Ehrenfest time. On the other hand, each matrix element in this basis includes a phase depending on the difference of actions between the corresponding periodic orbits [22]. So, to get sta- tistical independence between the perturbed and unperturbed eigenfunction sets, the perturbation has to modify the action of the used periodic orbits by at least $\hbar$, in a more or less random way. Specifically, the set of numbers $\left[\Delta S_{\mu} /(2 \pi \hbar)\right](\bmod$ 1 ), where $\mu$ labels periodic orbits with period shorter than the Ehrenfest time, has to be distributed uniformly in the interval $[0,1) ; \Delta S_{\mu}$ is the action of the unperturbed periodic orbit, $\mu$, minus the corresponding action in the perturbed case.

In conclusion, our results demonstrate that quantum systems with classically chaotic dynamics react in a universal way as a consequence of perturbations of classical nature. Specifically, the LDOS is a Breit-Wigner distribution, even for strong perturbations. Moreover, we derive a semiclassical expression for its width that is accurate for paradigmatic systems of quantum chaos as the cat maps and the stadium billiard. As a final remark, we would like to notice that our semiclassical result reproduces in part the old one obtained by Wigner [1] within the random matrix theory [23]. This fact implies that the connection between chaotic systems and random matrix theory, uncovered by the cellebrated BohigasGiannoni-Schmit conjecture [24], is stronger than believed.

We acknowledge the support from CONICET (PIP-112200801-01132), UBACyT (X237), ANPCyT and MTM200914621. We would like to thank Doron Cohen and Marcel Novaes for useful discussions.
[1] E. P. Wigner. Ann. Math. 62, 584 (1955).

[2] V. V. Flambaum, A. A. Gribakina, G. F. Gribakin, and M. G. Kozlov. Phys. Rev. A. 50, 267 (1994).

[3] Y Fyodorov, O Chubykalo, F Izrailev and G Casati. Phys. Rev. Lett. 70, 1603 (1996).

[4] G. Casati , B.V. Chirikov, I. Guarneri, and M. Izrailev. Phys. Lett. A 223, 430 (1996).

[5] D. Cohen and E.J. Heller. Phys. Rev. Lett. 84, 2841 (2000).

[6] R.A. Jalabert and H.M. Pastawski. Phys. Rev. Lett. 86, 2490 (2001).

[7] T. Gorin, T Prosen, TH Seligman and M. Z̆nidarič. Phys. Rep. 435, 33 (2006).

[8] Ph. Jacquod and C. Petitjean. . Adv. in Phys. 58, 67 (2009).

[9] D.A. Wisniacki and D. Cohen. Phys. Rev. E 66, 046209 (2002).

[10] S. M. Hammel, J. A. Yorke and C. Grebogi. J. Complex. 3, 136 (1987).

[11] J.Vanícek. Phys. Rev. E 70, 055201 (R) (2004); ibid 73, 046204 (2006).

[12] A.Goussev, D. Waltner, K. Richter and R. A. Jalabert. New Journal of Physics 10, 093010 (2008).

[13] M.Basilio De Matos, A. M. Ozorio De Almeida. Ann. Phys. 237, 46-65 (1995).
[14] N. Ares and D. A. Wisniacki. Phys. Rev. E 80, 046216 (2009).

[15] L. A. Bunimovich. Funct. Anal. Appl. 8254 (1974).

[16] D. A. Wisniacki and E. Vergini. Phys. Rev. E 59, 6579 (1999).

[17] N. Chernov. J. Stat. Phys. 88, 1 (1997).

[18] E. Vergini and M. Saraceno. Phys. Rev. E 52, 2204 (1995).

[19] A. H. Barnett, D. Cohen, and E. J. Heller. Phys. Rev. Lett. 85, 1412 (2000).

[20] E. G. Vergini. J. Phys. A: Math. and Gen. 33, 4709 (2000); E. G. Vergini, D. Schneider and A. M. F. Rivas. J. Phys. A: Math. Theor. 41, 405102 (2008).

[21] E. G. Vergini and G. G. Carlo. J. Phys. A: Math. and Gen. 34, 4525 (2001).

[22] E. G. Vergini and D. Schneider. J.Phys. A: Math. and Gen. 38, 587 (2005).

[23] In the limit of infinite perturbations, Wigner find a transition from the Lorentzian shape to a semicircular law. We speculate that our development is unable to detect such a transition because the used perturbations, even strong at quantum level, do not affect considerably the classical structure of the system.

[24] O. Bohigas, M. J. Giannoni and C. Schmit. Phys. Rev. Lett. 52, 1 (1984). 